\title{
Prospective validation of patient fatigue questionnaire (FACIT-F) for fatigue assessment in nab-paclitaxel plus gemcitabine therapy
}

\author{
KEN-ICHI OKADA $^{1}$, SEIKO HIRONO ${ }^{1}$, MANABU KAWAI $^{1}$, MOTOKI MIYAZAWA ${ }^{1}$, ATSUSHI SHIMIZU ${ }^{1}$, \\ YUJI KITAHATA $^{1}$, MASAKI UENO ${ }^{1}$, SHINYA HAYAMI ${ }^{1}$, TOSHIO SHIMOKAWA ${ }^{2}$ and HIROKI YAMAUE ${ }^{1}$ \\ ${ }^{1}$ Second Department of Surgery, ${ }^{2}$ Department of Clinical Study Support Center, \\ Wakayama Medical University, Wakayama, Wakayama 641-8510, Japan
}

Received May 21, 2017; Accepted October 4, 2017

DOI: $10.3892 / \mathrm{mco} .2017 .1485$

\begin{abstract}
Following the recent introduction of a stronger regimen for pancreatic carcinoma, patients undergoing chemotherapy present high incidences of fatigue. The feasibility and validity of evaluating fatigue by questionnaire for patients with unresectable pancreatic cancer (URPC) receiving this recent regimen of chemotherapy remains unclear. Enrolled patients completed the Functional Assessment of Cancer Therapy-Fatigue questionnaire (FACIT-F, version 4), a questionnaire regarding additional concerns, a numerical rating scale test concerning loss of appetite, an evaluation of degree of pain and sensory disorder, and the Patient Neurotoxicity Questionnaire (PNQ) before undergoing nab-paclitaxel plus gemcitabine therapy. Questionnaires and tests were completed on registration day, and then weekly during therapy over an 8 -week period as the initial two cycles of continuous regimen. This trial is registered on the UMIN Clinical Trials Registry (UMIN000021758). Between April 2016 and September 2016, 10 consecutive patients with URPC, including metastatic $(n=4)$ and locally advanced pancreatic $(n=6)$ cancer, were registered, and scheduled for nab-paclitaxel plus gemcitabine therapy. The mean maximum values of fatigue degree increased from mean baseline values in all categories of the questionnaire (6-500\%). The degree of fatigue presented a spike pattern over a 4-week scheduled period as one cycle of regimen in time-sequence diagram regarding 10/13 (77\%) questionnaires. The PNQ concerning sensory/motor disorder also demonstrated a spike pattern and an increase from the baseline as the number of administrations. It is valid and feasible to assess fatigue by FACIT-F questionnaire for patients with URPC undergoing nab-paclitaxel plus gemcitabine therapy,
\end{abstract}

Correspondence to: Dr Hiroki Yamaue, Second Department of Surgery, Wakayama Medical University, 811-1 Kimiidera, Wakayama, Wakayama 641-8510, Japan

E-mail: yamaue-h@wakayama-med.ac.jp

Key words: fatigue, pancreatic carcinoma, chemotherapy, patient-reported outcomes, nab-paclitaxel, gemcitabine and to detect detailed changes in accordance with scheduled cycles of chemotherapy regimen.

\section{Introduction}

Pancreatic cancer is projected to surpass breast, prostate, and colorectal cancers to become the second leading cause of cancer-related death by 2030. It is associated with extremely poor prognosis $(1,2)$. Patients with advanced pancreatic carcinoma usually present some extent of accompanying fatigue. After the introduction of recent stronger regimen of chemotherapy, patients presented a high incidence of fatigue $(3,4)$. Fatigue impairs patients' physical and mental energy, directly and indirectly influencing the sustainability of chemotherapy. Nab-paclitaxel plus gemcitabine is a standard therapy and a promising treatment for unresectable pancreatic cancer (URPC) including metastatic/locally advanced pancreatic cancer (3). In an international multicenter open-label randomized phase 3 study, Von Hoff et al reported the considerable frequency of treatment-related adverse events rated grade 3 or higher of the nab-paclitaxel plus gemcitabine therapy as neutropenia (38\%), leucopenia (31\%), fatigue (17\%), and peripheral neuropathy (17\%) (3). Frequency and degree gradually rise, particularly during chemotherapy, significantly impacting on quality of life of URPC patients. Quality of life is easily impaired by fatigue and peripheral neuropathy in patients who receive nab-paclitaxel plus gemcitabine therapy (5). Patient-reported outcomes are useful for evaluating adverse effects that are difficult to assess objectively by physicians. Functional Assessment of Chronic Illness Therapy-Fatigue (FACIT-F) questionnaire $(6,7)$ and Patient Neurotoxicity Questionnaire (PNQ) (8) have been utilized to assess patient-reported outcomes of cancer treatments such as chemotherapy or chemoradiotherapy to adjust to sustainable dosage of agents. However, the feasibility and sensitivity of fatigue assessment in patients receiving recent stronger chemotherapy regimen is unknown.

We prospectively investigated the feasibility and validity of a patient-based scale, FACIT-F Questionnaire, PNQ, toxicities, and adverse effects evaluated in accordance with Common Terminology Criteria for Adverse Events (CTCAE) version 4.0 (9) for cumulative fatigue and peripheral neuropathy in the timing of the introduction of nab-paclitaxel plus gemcitabine 
therapy. The data from this study could be useful for identifying appropriate timing and duration of medical intervention for supporting therapy in nab-paclitaxel plus gemcitabine therapy.

\section{Patients and methods}

Patients. The present study was approved by the Wakayama Medical University Hospital Institutional Review Board (approval no. 1771). This trial is registered on the UMIN Clinical Trials Registry (UMIN000021758). We excluded patients with severe comorbidity, such as severe cardiac/renal failure or bowel obstruction and those unable to intake oral medicine, to facilitate comparison of data with the next interventional trial (UMIN000025606) where endpoints are defined identically to this study. Criteria of eligible patients enrolled in this study were as follows: Patients with URPC who received nab-paclitaxel plus gemcitabine therapy as a first line chemotherapy and had an Eastern Cooperative Oncology Group (ECOG) PS of 0 or 1; $\geq 20$ years old. Written informed consent to participate in this study was also required. Additionally, the following criteria had to be satisfied in laboratory tests within 14 days of registration: WBC count $\geq 3,500 / \mathrm{mm}^{3}$ and $\leq 12,000 / \mathrm{mm}^{3}$, neutrophil count $\geq 1,500 / \mathrm{mm}^{3}, \mathrm{Hb} \geq 9.0 \mathrm{~g} / \mathrm{dl}, \mathrm{Plt} \geq 100,000 / \mathrm{mm}^{3}, \mathrm{~T}$. Bil $\leq 2.0 \mathrm{mg} / \mathrm{dl}$ ( $\leq 3.0 \mathrm{mg} / \mathrm{dl}$ in biliary drainage case), serum $\mathrm{Cr} \leq 1.5 \mathrm{mg} / \mathrm{dl}$, and AST, ALT $\leq 100 \mathrm{IU} / \mathrm{l}$. Patients with URPC during the period of this study received nab-paclitaxel plus gemcitabine therapy as a first line chemotherapy in our institute. In this study, locally advanced pancreatic cancer was defined according to National Comprehensive Cancer Network (NCCN) version 2.2016 criteria (10).

Endpoints. The primary endpoint of this study was to investigate the feasibility and validity of fatigue evaluation by the FACIT-F version 4 questionnaire and additional concerns (Japanese version) for URPC patients. Secondary endpoints included: Appetite loss, degree of pain and sensory disorder evaluated by Numerical Rating Scale (NRS), cumulative sensory/motor neurotoxicity with Patient Neurotoxicity Questionnaire (PNQ).

Treatment. Enrolled patients were administered a 30-min intravenous infusion of nab-paclitaxel at a dose of $125 \mathrm{mg} / \mathrm{m}^{2}$, followed by a 30-min intravenous infusion of gemcitabine at a dose of $1,000 \mathrm{mg} / \mathrm{m}^{2}$, on days 1,8 and 15 over a four-week period as one cycle of regimen similar to that previously reported (5). There was one week of rest between each cycle. The criteria for restart, dose reduction, and discontinuation of chemotherapy were also as previously reported (5). Treatment was repeated until disease progression or toxicity levels became unacceptable, or when discontinuation was decided by the investigators or by patient refusal. In the absence of disease progression, patients continued chemotherapy.

Assessments. Enrolled patients completed FACIT-F (version 4) and questionnaires about additional concerns, an NRS test about appetite loss, degree of pain and sensory disorder (cold, burning) and PNQ before administration of nab-paclitaxel plus gemcitabine. Questionnaires and tests were completed on registration day and weekly thereafter during therapy on days
Table I. Characteristics of patients.

\begin{tabular}{|c|c|}
\hline Characteristic $^{\mathrm{a}}$ & Value \\
\hline \multicolumn{2}{|l|}{ Baseline } \\
\hline Sex (male/female) & $5 / 5$ \\
\hline Age, years & $63 \pm 10.3$ \\
\hline Location of pancreatic cancer (body-tail/head) & $4 / 6$ \\
\hline \multicolumn{2}{|l|}{ Comorbidity, patient no. } \\
\hline Diabetes mellitus & 3 \\
\hline Hypertension & 1 \\
\hline Biliary stent or drainage & 6 \\
\hline \multicolumn{2}{|l|}{ Performance status } \\
\hline 0 & 10 \\
\hline 1 & 0 \\
\hline 2 & 0 \\
\hline 3 & 0 \\
\hline 4 & 0 \\
\hline Frequency of administration & $5.0 \pm 0.9$ \\
\hline Skipping of administration & $1.0 \pm 0.9$ \\
\hline Dose reduction & 5 \\
\hline Metastatic/locally advanced & $4 / 6$ \\
\hline \multicolumn{2}{|l|}{ UICC stage } \\
\hline IIA & 1 \\
\hline IIB & 0 \\
\hline III & 5 \\
\hline IV & 4 \\
\hline Discontinuation of chemotherapy & 0 \\
\hline \multicolumn{2}{|l|}{ Response evaluation } \\
\hline Partial response & 1 \\
\hline Stable disease & 8 \\
\hline Progressive disease & 1 \\
\hline Decrease rate of CA 19-9 value & $0.50 \pm 0.5$ \\
\hline
\end{tabular}

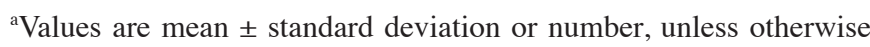
stated. UICC, Union for International Cancer Control; CA 19-9, cancer antigen 19-9.

$1,8,15,22,29,36,43$ and 50 over an eight-week period as the first two cycles of continuous regimen. FACIT-F was evaluated by degree and each degree was converted to numerical values as follows: 0: Not at all; 1: A little bit; 2: Somewhat; 3: Quite a bit; 4: Very much. Total values were recorded weekly for each questionnaire. Appetite loss, degree of pain and sensory disorder were evaluated by NRS converting to 0-10, cumulative sensory/motor neurotoxicity with PNQ converting to 0-4.

Toxicities and adverse effects of chemotherapy were evaluated in accordance with Common Terminology Criteria for Adverse Events [CTCAE] version 4.0. Complete blood counts and differential count of leukocytes, blood chemical tests and physical examinations were carried out at least once per week until the end of the two cycles and every two weeks thereafter. In cases of grade 4 hematological toxicity, re-examination within four days was required. We carried out computed tomography when the tumor marker was extremely elevated. 
Table II. FACIT-F (version 4) questionnaire additional concerns.

\begin{tabular}{|c|c|c|c|}
\hline Question & $\begin{array}{l}\text { Baseline } \\
\text { (Degree) }\end{array}$ & $\begin{array}{c}\text { Maximum of mean } \\
\text { (Mean) }\end{array}$ & Increase rate $(\%)$ \\
\hline \multicolumn{4}{|l|}{ HI7 } \\
\hline I feel fatigued & $1.4(0-2)$ & $1.9(1.3-1.9)$ & 36 \\
\hline \multicolumn{4}{|l|}{ HI12 } \\
\hline I feel weak all over & $1.8(0-4)$ & $1.9(1.6-1.9)$ & 6 \\
\hline \multicolumn{4}{|l|}{ An1 } \\
\hline I feel listless ('washed out') & $0.9(0-2)$ & $1.5(0.7-1.5)$ & 67 \\
\hline \multicolumn{4}{|l|}{ An2 } \\
\hline I feel tired & $1.6(1-3)$ & $1.9(1.1-1.9)$ & 19 \\
\hline \multicolumn{4}{|l|}{ An3 } \\
\hline I have trouble starting things because I am tired & $1.2(0-3)$ & $1.6(0.6-1.6)$ & 33 \\
\hline \multicolumn{4}{|l|}{ An 4} \\
\hline I have trouble finishing things because I am tired & $1.0(0-3)$ & $1.5(0.6-1.5)$ & 50 \\
\hline \multicolumn{4}{|l|}{ An5 } \\
\hline I have energy & $1.6(0-2)$ & $2.3(1.6-2.3)$ & 44 \\
\hline \multicolumn{4}{|l|}{ An7 } \\
\hline I am able to do my usual activities & $2.3(0-4)$ & $2.9(2.1-2.9)$ & 26 \\
\hline \multicolumn{4}{|l|}{ An8 } \\
\hline I need to sleep during the day & $1.2(0-2)$ & $1.8(1.0-1.8)$ & 50 \\
\hline \multicolumn{4}{|l|}{ An12 } \\
\hline I am too tired to eat & $0.2(0-1)$ & $1.2(0.4-1.2)$ & 500 \\
\hline \multicolumn{4}{|l|}{ An14 } \\
\hline I need help doing my usual activities & $0.5(0-2)$ & $1.1(0.3-1.1)$ & 120 \\
\hline \multicolumn{4}{|l|}{ An15 } \\
\hline I am frustrated by being too tired to do the things I want to do & $0.5(0-2)$ & $1.2(0.4-1.2)$ & 140 \\
\hline \multicolumn{4}{|l|}{ An16 } \\
\hline I have to limit my social activity because I am tired & $1.0(0-3)$ & $1.6(0.8-1.6)$ & 60 \\
\hline
\end{tabular}

Values are mean of degree. Degree: 0, Not at all; 1, A little bit; 2, Somewhat; 3, Quite a bit; 4, Very much. FACIT-F, Functional Assessment of Cancer Therapy-Fatigue.

Tumor response was reviewed in accordance with Response Evaluation Criteria in Solid Tumors (RECIST) version 1.1.

Statistical analysis. We evaluated the total score of questionnaires using linear-mixed effect modeling with patients as a random effect and treatment courses and weeks as fixed effects.

\section{Results}

Patient characteristics. Between April 2016 and September 2016, 10 consecutive patients with unresectable pancreatic cancer (URPC) including metastatic $(n=4) /$ locally advanced pancreatic $(n=6)$ cancer were registered and scheduled for nab-paclitaxel plus gemcitabine therapy. Baseline compliance with completion of FACIT-F, NRS, and PNQ revealed no data deficit: $100 \%$ were completed in all questionnaires or tests from all the patients. Table I shows the characteristics of the analyzed patients. No patients in this study discontinued chemotherapy.

Endpoints. Table II shows patient-reported outcomes by means of FACIT-F fatigue evaluation in the present study.
The total of each mean value of the questionnaire also showed spike patterns in the time-sequence diagram (Fig. 1A) and the fluctuation range based on the maximum and minimum values was 7.3 (12.9-20.2), an increased rate from baseline $35 \%$. The value of the first day of the second course was elevated compared to that of the first course.

Table III shows the results of the mixed effect model. There were no significant differences in each of the fixed effects. However, fatigue evaluated by CTCAE revealed increase only in the number of patients who were evaluated as all grade fatigue without any patterns (Fig. 1B). The mean maximum values of fatigue degree increased from mean baseline values in all categories of questionnaire (6-500\%). In addition, the degree of fatigue shows a spike pattern over a four-week scheduled period as one cycle of regimen in time-sequence diagram regarding ten of thirteen (77\%) questionnaires (HI12, An1, An2, An3, An4, An8, An12, An14, An15, An16) (Fig. 2). Secondary endpoints did not reveal any specific patterns in appetite loss, but the degree of pain and sensory disorder evaluated by NRS revealed a spike pattern in the number of patients (data not shown). PNQ concerning sensory/motor 
Table III. Results of mixed effect model.

\begin{tabular}{lcc}
\hline Fixed effect & P-value & Coefficients \\
\hline Courses & 0.299 & 1.55 \\
Weeks & 0.137 & 1.04 \\
\hline
\end{tabular}
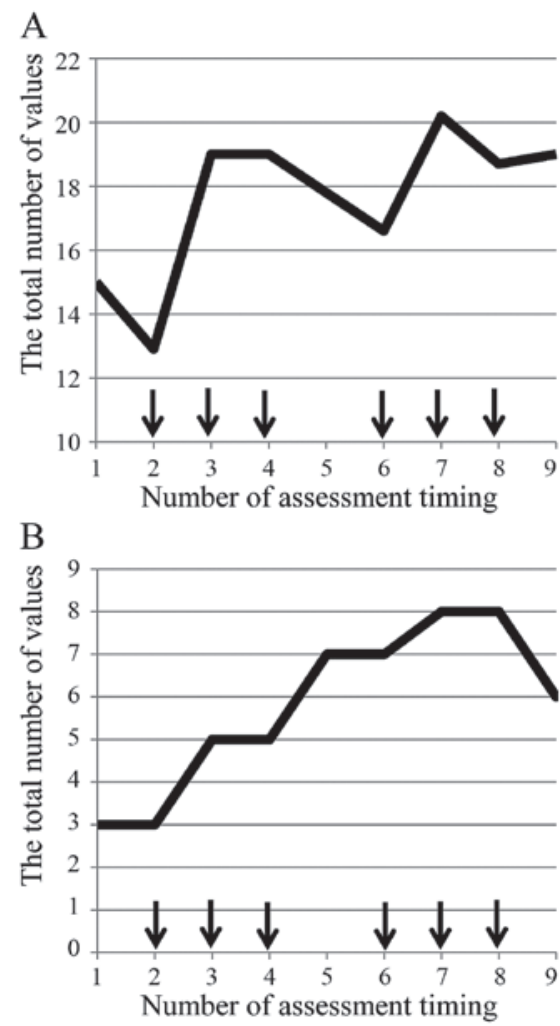

Figure 1. (A) FACIT-F was evaluated by the mean of degree, and each degree was converted to numerical values as follows: 0 : Not at all; 1 : A little bit; 2 : Somewhat; 3: Quite a bit; 4: Very much. Total values were recorded weekly for each questionnaire. The total value of means in each questionnaire show a spike pattern in time-sequence diagram. (B) Fatigue evaluated by CTCAE revealed only an increase in the number of patients who were evaluated as all grade fatigue without showing any patterns. Enrolled patients were administered intravenous infusion of nab-paclitaxel, followed by intravenous infusion of gemcitabine with two cycles, on days 1,8 and 15 over a four-week period as one cycle of regimen (arrow). Vertical lines show the total number of values assessed by each assessment tool, and horizontal lines represent the number of assessment timing, i.e., 1-9, on registration day, and weekly assessment during therapy on days $1,8,15,22,29,36,43$ and 50 over an eight-week period as first two cycles of consecutive regimen.

disorder demonstrated a spike pattern and increase from the baseline as the number of administrations (Fig. 3). No patients presented burning pain, but the incidence of cold sensory disorder increased with the number of administrations (Fig. 4).

Toxicities and adverse effects. Adverse drug reactions deemed to be potentially related to the nab-paclitaxel plus gemcitabine therapy are shown in Table IV. The overall rate of any grade events (CTCAE ver. 4.0 criteria) during the treatment was $100 \%$. The overall rate of patients who presented grade 3 and 4 events was $80 \%$. The majority of these adverse events represented leucopenia $(80 \%)$, appetite loss $(80 \%)$, hair loss
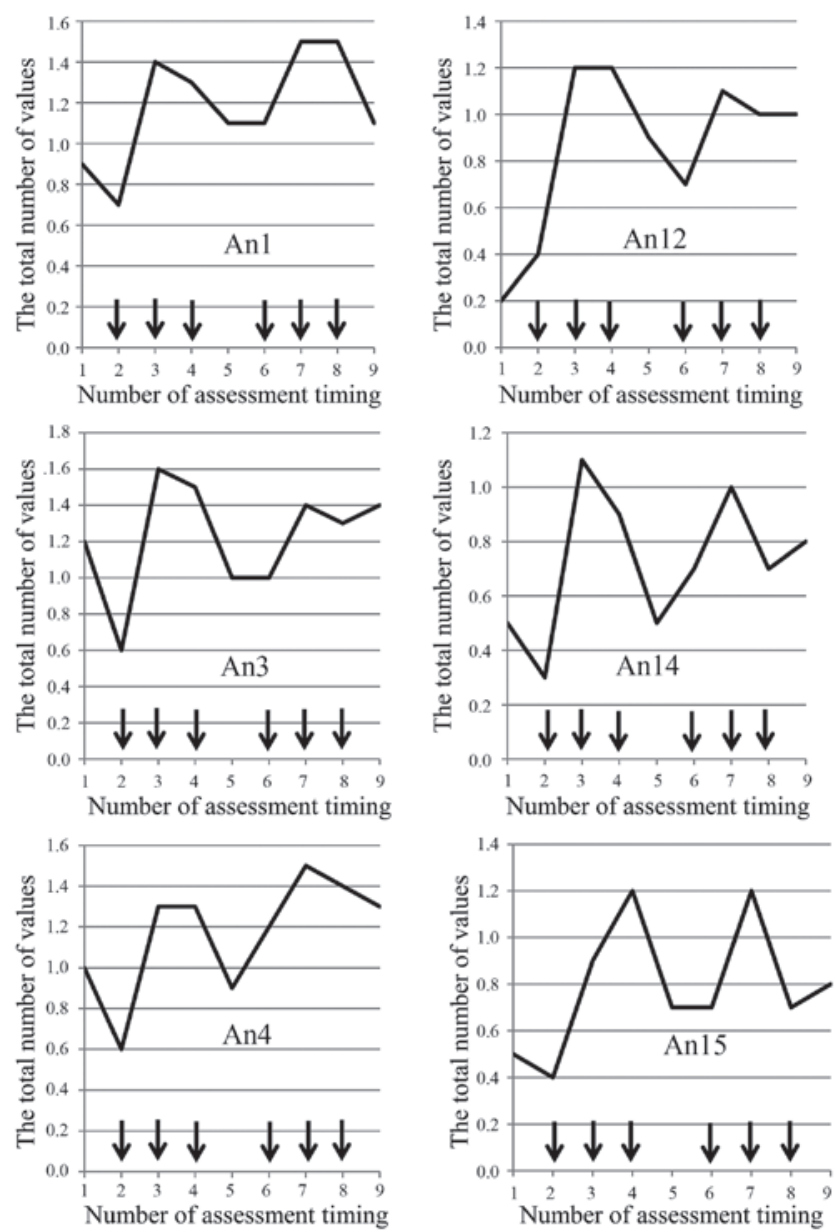

Figure 2. FACIT-F was evaluated by mean of degree, and each degree was converted to numerical values as follows: 0: Not at all; 1: A little bit; 2: Somewhat; 3: Quite a bit; 4: Very much. Total values were recorded weekly for each questionnaire. The degree of fatigue shows a spike pattern over a four-week scheduled period as one cycle of regimen in time-sequence diagram. Graphical data regarding question numbers An1, An3, An4, An12, An14, An15 are shown.

(90\%), and fatigue (90\%). There were no incidences of serious adverse events such as febrile neutropenia, sepsis, grade three or higher interstitial pneumonia, and no treatment-related deaths in this study (Table IV).

\section{Discussion}

Fatigue is a common lasting symptom in most patients who receive chemotherapy, its control is a key to the sustainability of chemotherapy treatment. Although fatigue affects quality of life in patients with advanced pancreatic cancer, detailed change of fatigue levels during chemotherapy remains to be investigated. In the present study, we prospectively demonstrated the feasibility and validity of the FACIT-F Questionnaire, and its detailed change in accordance with scheduled cycles of chemotherapy regimen. Compared with CTCAE assessment of fatigue, FACIT-F reflects spike patterns in the degree of fatigue in phases with each cycle of regimen. The presence and its amplitude revealed the chance of intervention for fatigue. Until now, there were few effective established preventive measures in the field of supportive care medicine. Unfortunately, the total score of the questionnaire revealed no significant increase in using linear-mixed effect modeling analyses regarding 
Table IV. Toxicity following treatment with neoadjuvant nab-paclitaxel plus gemcitabine therapy.

\begin{tabular}{lccc}
\hline Treatment toxicity & All grade & G3 & G4 \\
\hline Leucopenia & $8(80)$ & $5(50)$ & 0 \\
Anemia & $1(10)$ & 0 & 0 \\
Thrombocytopenia & $4(40)$ & $1(10)$ & 0 \\
Neutropenia & $5(50)$ & $3(30)$ & $1(10)$ \\
Liver dysfunction & $2(20)$ & 0 & 0 \\
Appetite loss & $8(80)$ & 0 & 0 \\
Nausea & $6(60)$ & 0 & 0 \\
Vomit & $1(10)$ & 0 & 0 \\
Diarrhea & 0 & 0 & 0 \\
Fatigue & $9(90)$ & $2(20)$ & 0 \\
Oral inflammation & $3(30)$ & 0 & 0 \\
Hand foot syndrome & 0 & 0 & 0 \\
Hair loss & $9(90)$ & 0 & 0 \\
Febrile neutropenia & 0 & 0 & 0 \\
Cholangitis & 0 & 0 & 0 \\
Interstitial pneumonia & 0 & 0 & 0 \\
Peripheral sensory & & & \\
Neuropathy & $3(30)$ & 0 & 0 \\
\hline
\end{tabular}

Values are number of events (\%). Safety was evaluated in accordance with the Common Terminology Criteria for Adverse Events version 4.0

treatment courses and weeks as fixed effects. This could be due to the short observation duration of this study. We also observed other adverse effects such as appetite loss, degree of pain and sensory disorders, and cumulative sensory/motor neurotoxicity since the mechanism of fatigue and relationships with other adverse effects remains unknown.

This study demonstrated fatigue alternated between stronger and weaker in synch with an on- and-off chemotherapy schedule, but may be influenced by other adverse effects, such as the number of administrations. Recently, a website was designed to provide researchers information about Patient-Reported Outcomes version of the Common Terminology Criteria for Adverse Events (PRO-CTCAE ${ }^{\mathrm{TM}}$ ), a patient-reported outcome measurement system developed by the National Cancer Institute to capture symptomatic adverse events in patients in cancer clinical trials (11). Although we mainly intended to assess quality of life by FACIT-F and by referring to PRO-CTCAE recommendation, we designed a weekly assessment for the introductory period of chemotherapy in this study, i.e., weekly assessment during the first two cycles of consecutive chemotherapy to compare with CTCAE assessment about fatigue. The FACIT-F questionnaire is composed of five sections as follows: Well-being, social/family well-being, emotional well-being, functional well-being and additional concerns. We limited assessment of the additional concerns section by considering only appropriate questions directly reflecting patient's fatigue and the outcomes originating from fatigue. In addition, we avoided patient burden from high number of questions to a

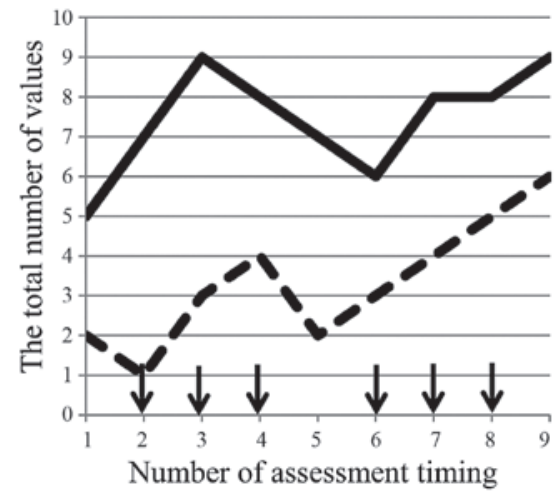

Figure 3. Patient Neurotoxicity Questionnaire of sensory (dotted line)/motor (solid line) disorder shows a spike pattern and increase from baseline as the number of administrations.

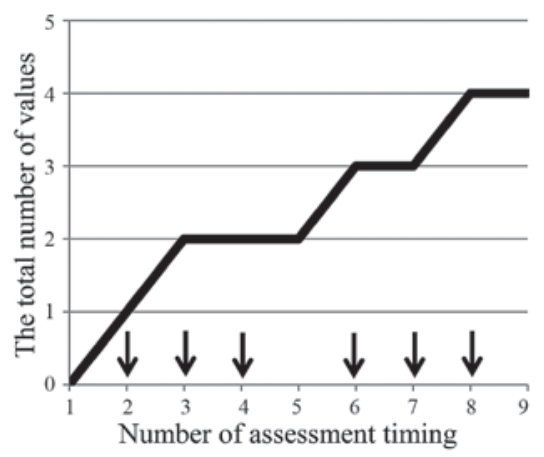

Figure 4. Cold sensory disorder increased in incidence with the number of administrations.

single assessment in this study requiring a short time (within $3 \mathrm{~min}$ ). However, this is also a major limitation of this study. The results of our study revealed the feasibility and validity of the FACIT-F questionnaire in accordance with the CTCAE assessment about fatigue in patients on cancer clinical trials. In addition to that, FACIT-F assessment revealed the elevated fatigue status during chemotherapy. Based on the results of this study, we have just started the next interventional trial (UMIN000025606) in which patients were administered Japanese herbal medicines for fatigue, and fatigue levels were assessed in a similar way. We aim to confirm, not only the feasibility and validity by means of the FACIT-F questionnaire in accordance with the CTCAE assessment, but also the differences between the two assessments about fatigue.

In conclusion, it was feasible to use the FACIT-F Questionnaire for assessment of patients with URPC who underwent nab-paclitaxel plus gemcitabine therapy, and to detect detailed changes in accordance with scheduled cycle of chemotherapy regimen. The data obtained from this tool are useful for identifying the timing and duration of medical intervention for supporting therapy in cancer patients.

\section{Acknowledgements}

We would like to thank the Department of Clinical Study Support Center, Wakayama Medical University, for proofreading and editing the manuscript. 


\section{References}

1. Siegel RL, Miller KD and Jemal A: Cancer statistics, 2016. CA Cancer J Clin 66: 7-30, 2016.

2. Rahib L, Smith BD, Aizenberg R, Rosenzweig AB, Fleshman JM and Matrisian LM: Projecting cancer incidence and deaths to 2030: The unexpected burden of thyroid, liver, and pancreas cancers in the United States. Cancer Res 74: 2913-2921, 2014.

3. Von Hoff DD, Ervin T, Arena FP, Chiorean EG, Infante J, Moore M, Seay T, Tjulandin SA, Ma WW, Saleh MN, et al: Increased survival in pancreatic cancer with nab-paclitaxel plus gemcitabine. N Engl J Med 369: 1691-1703, 2013.

4. Conroy T, Desseigne F, Ychou M, Bouché O, Guimbaud R, Bécouarn Y, Adenis A, Raoul JL, Gourgou-Bourgade S, de la Fouchardière $\mathrm{C}$, et al: FOLFIRINOX versus gemcitabine for metastatic pancreatic cancer. N Engl J Med 364: 1817-1825, 2011.

5. Okada KI, Hirono S, Kawai M, Miyazawa M, Shimizu A, Kitahata Y, Ueno M, Hayami S and Yamaue H: Phase I study of nab-paclitaxel plus gemcitabine as neoadjuvant therapy for borderline resectable pancreatic cancer. Anticancer Res 37: 853-858, 2017.

6. Salsman JM, Beaumont JL, Wortman K, Yan Y, Friend J and Cella D: Brief versions of the FACIT-fatigue and FAACT subscales for patients with non-small cell lung cancer cachexia. Support Care Cancer 23: 1355-1364, 2015.
7. Younossi ZM, Stepanova M, Charlton M, Curry MP, O'Leary JG Brown RS and Hunt S: Patient-reported outcomes with sofosbuvir and velpatasvir with or without ribavirin for hepatitis $C$ virus-related decompensated cirrhosis: An exploratory analysis from the randomised, open-label ASTRAL-4 phase 3 trial. Lancet Gastroenterol Hepatol 1: 122-132, 2016.

8. Shimozuma K, Ohashi Y, Takeuchi A, Aranishi T, Morita S, Kuroi K, Ohsumi S, Makino H, Mukai H, Katsumata N, et al: Feasibility and validity of the Patient Neurotoxicity Questionnaire during taxane chemotherapy in a phase III randomized trial in patients with breast cancer: N-SAS BC 02. Support Care Cancer 17: 1483-1491, 2009.

9. National Cancer Institute: Common terminology criteria for adverse events (CTCAE) Version 4.0. https://evs.nci.nih. gov/ftp1/CTCAE/CTCAE_4.03_2010-06-14_QuickReference 5x7.pdf. Accessed March 3, $201 \overline{7}$.

10. National Comprehensive Cancer Network: NCCN practice guidelines for pancreatic cancer, version 2,2016. http://www.ncen. org/professionals/physician_gls/PDF/pancreatic.pdf. Accessed October 4, 2016.

11. National Cancer Institute:Patient-reported outcomes version of the common terminology criteria for adverse events (PRO-CTCAE ${ }^{\mathrm{TM}}$ ). https://healthcaredelivery.cancer.gov/ pro-ctcae/pro-ctcae_japanese.pdf\#search=pro-ctcae. Accessed March 3, 2017. 